Article

\title{
Polygenetic Variants Related to Osteoarthritis Risk and Their Interactions with Energy, Protein, Fat, and Alcohol Intake in Adults in a Large Cohort
}

\author{
Sunmin Park
}

check for updates

Citation: Park, S. Polygenetic Variants Related to Osteoarthritis Risk and Their Interactions with Energy, Protein, Fat, and Alcohol Intake in Adults in a Large Cohort. Diagnostics 2022, 12, 340. https:/ / doi.org/10.3390/diagnostics12020340 Academic Editor: Evangelos Terpos Received: 29 December 2021 Accepted: 27 January 2022 Published: 28 January 2022

Publisher's Note: MDPI stays neutral with regard to jurisdictional claims in published maps and institutional affiliations.

Copyright: (C) 2022 by the author. Licensee MDPI, Basel, Switzerland. This article is an open access article distributed under the terms and conditions of the Creative Commons Attribution (CC BY) license (https:// creativecommons.org/licenses/by/ $4.0 /)$.
Food and Nutrition, Obesity/Diabetes Center, Hoseo University, 165 Sechul-Ri, BaeBang-Yup, Asan-Si 31499, Korea; smpark@hoseo.edu; Tel.: +82-41-540-5345; Fax: +82-41-548-0670

\begin{abstract}
Osteoarthritis (OA) is increasing globally, especially among elderly Asian women, and its increase may be due to the interaction between genetic factors and lifestyle. This study tested the hypothesis that polygenetic variants associated with OA risk interacted with lifestyle in adults over 40 years in the Ansan-Ansung cohort. Genetic variants were chosen through a genome-wide association study with OA participants (case; $n=580)$ and controls without arthritis $(n=4850)$. Genetic variants with interactions were selected by a generalized multifactor dimensionality reduction. The best model's polygenic risk scores (PRS) were calculated by summing the number of risk alleles in the selected genetic variants. The best five single nucleotide polymorphism (SNP) model included AIG1_rs6570550, COX10_rs62054459,DLG2_rs148643344, SOX5_rs73283615, and PLXNA4_rs1472529430, while IL12A_rs1491318751 was added to the five-SNP model to produce a six-SNP model. Only COX10_rs62054459 in subcutaneous and visceral adipose tissue was associated with COX10 protein expression. The participants, having high-PRS from the five-SNP and six-SNP models, were at a higher OA risk than those with low-PRS by 3.88 and 4.42 times, respectively. The PRS was not associated with metabolic syndrome or with the insulin resistance index (HOMA-IR). Energy, protein, fat, alcohol, and a Western-style diet intake interacted with the PRS to influence OA risk ( $p=0.005,0.042$, and 0.021 , respectively). In the high energy and alcohol intake and low protein, fat, Western-style diet intake, the participants with a high-PRS had a higher incidence of OA than those with low-PRS. In conclusion, the adults with a high-PRS were at a higher OA risk. Particularly, adults with high PRS should have a lower energy intake, higher WSD containing higher protein and fat intake, and moderate alcohol intake to alleviate OA risk. These results can be applied to personalized nutrition plans to decrease OA risk.
\end{abstract}

Keywords: osteoarthritis; immune-related diseases; inflammation; energy; protein; genetic variants

\section{Introduction}

The prevalence of degenerative diseases is increasing as the aging population is increasing worldwide. Osteoarthritis (OA) is a degenerative disease that worsens over time and is the most prevalent form of arthritis [1]. OA is a leading cause of disability in older adults. According to the World Health Organization (WHO), the prevalence of OA globally is approximately $9.6 \%$ and $18.0 \%$ in men and women, respectively. It is increasing rapidly in Asians because the elderly Asian population has increased rapidly from $6.8 \%$ in 2008 and is predicted to increase to $16.2 \%$ in 2040 [1].

OA is a 'wear and tear' disease with chronic pain and inflammation in the joints. The disease is induced by progressive damage to articular cartilage and bone remodeling and is worsened by synovial inflammation, fibrosis of ligaments and tendons, and thickening of the capsules. OA is a multifactorial and complex disease. The unmodifiable risk factors involved in OA are older age, women, and genetics, while the modifiable risk factors are obesity, type 2 diabetes, joint injuries, repeated physical stress on the joints by work and 
exercise, alcohol consumption, and dietary intake [2-4]. Heavy occupational, physically active, and agricultural work increase symptomatic knee and hip OA. Dietary factors influence OA risk by modulating inflammation, oxidative stress, and weight $[3,4]$. Obesity is a primary risk factor for OA in Western countries, but it is not confirmed in Asians, who are generally not severely obese. Since obesity rates have increased in Asians, obesity will become an essential risk factor in Asia in the future.

Asians generally have a lower risk of OA in most joints, except for the knee joints, than Caucasians [5]. Along with modifiable environmental factors, genetic factors contribute to OA development. Genetic association studies have identified the effects of critical genetic variants on $\mathrm{OA}$ pathogenesis. On the other hand, each significant genetic variant has minor impacts, indicating that complex polygenic variants influence OA etiology [2]. Although genetic factors along with age and gender are unmodifiable factors, they interact with lifestyle to influence OA risk. Environmental factors, including lifestyle, interact with genetic factors, and can be applied to personalized nutrition to prevent and manage OA.

Genome-wide linkage scans have identified OA susceptibility genes, such as chromosomes 2, 3, 4, 6, 7, 11, 16, and X. Chromosome 2q13-32 includes the interleukin-1 (IL-1) gene cluster, frizzled-related protein $3(F R Z B)$, and cartilage structural protein matrilin-3 (MATN3) and is associated with OA risk. Other genes related to OA are alpha1 type IX collagen (COL9A1), bone morphogenetic protein 5 (BMP5), IL-4R, and low-density lipoprotein receptor-related protein 5 (LRP5) genes [2]. Similar to the linkage gene study, Gly976Ser and Arg519Cys variations of the Collagen Type II Alpha 1 Chain (COL2A1), the primary components of articular cartilage and intervertebral discs, are associated with knee and hip $\mathrm{OA}$ risk and reduce the durability of the articular cartilage against mechanical stress [6]. Moreover, the AA genotype of COL2A1 single-nucleotide polymorphism (SNP) G4006A is positively associated with OA risk, while the TA genotype of T2088C and G4006A haplotype is a positive risk factor for OA in Han Chinese women [7]. On the other hand, few studies on the genetic impact of OA and the interaction between genetic and environmental factors, have been conducted in Koreans. Some potential interactions between OA genetic risk and environmental factors have been reported. Physical activity can modulate gut microbiota composition to promote intestinal mucosal immunity, modify the bile acid profile, and improve the production of short-chain fatty acids by activating a gut-joint axis [8].

This study tested the hypothesis that complex polygenetic variants influenced OA risk and the genetic impact interacted with the lifestyles of Asian adults. This hypothesis was assessed in adults over 40 years in the Ansan-Ansung cohort in Korea. Identifying novel genetic variants and their interaction with lifestyle will eventually provide a better understanding of the OA molecular mechanism, particularly for Asians, and a potential personalized dietary regime for OA prevention and management in the future.

\section{Methods}

\subsection{Participants}

Data collected from 2008-2014 for the Korean Genome and Epidemiology Study (KoGES) were used [9]. Participants were recruited from the rural community of Ansung and the urban community of Ansan city, where they must have resided for at least six months. A total of 5430 participants aged over 40 years (2589 men and 2841 women) were included. All procedures of the KoGES followed the Declaration of Helsinki and were approved by the Institutional Review Boards of the Korean National Institute of Health (1041231-190902-BR-099-01) and Hoseo University (1041231-150811-HR-034-01). Written informed consent was obtained from all participants.

\subsection{General Characteristics and Biochemical Measurements}

The participants were mentally and physically healthy. Age, gender, education, income, physical activity, smoking status, and alcohol consumption were collected during a health interview. Body weight, height, fat, and skeletal muscle mass were measured 
using Inbody (Inbody 3.0, Biospace, Cheonan, Korea) wearing a light gown [10]. The waist circumference was also measured, $2 \mathrm{~cm}$ above the navel, around the waist with a tape measure. The body mass index (BMI) was calculated by dividing the weight (kg) by the square height $\left(\mathrm{m}^{2}\right)$.

Education level was categorized into: less than high school, high school, and college or more. Household income (USD/month) was divided into four groups: very low $(<1000)$, low (1000-2000), intermediate (2000-4000), and high (>4000). Smoking status was categorized into: current smoker, past smoker, and never smoker. A current smoker was defined as having smoked more than 100 cigarettes over the previous six months [11]. Alcohol consumption was assessed by questioning the participants about their drinking behavior during the month before the interview. According to average daily consumption (g/day), the alcohol consumption status was divided into nondrinker, light drinker (1-15), moderate drinker (16-30), and heavy drinker (>30).

Blood pressure was measured on the right arm, in a sitting position, at heart level. Biochemical parameters were determined using plasma and serum from blood drawn after an overnight fast (no foods for more than $12 \mathrm{~h}$ ) [12]. The lipid profile (total cholesterol, HDL cholesterol, and triglyceride), glucose, aspartate aminotransferase (AST), alanine aminotransferase (ALT), and creatinine concentrations in the circulation were measured using a Hitachi 7600 Automatic Analyzer (Hitachi Ltd., Tokyo, Japan). The white blood cell (WBC) counts and hemoglobin A1c (HbA1c; glycated hemoglobin) concentrations in heparin-treated blood were determined using a Hitachi 7600 Automatic Analyzer (Hitachi, Tokyo, Japan). Serum high-sensitive C-reactive protein (hs-CRP) and insulin concentrations were measured using an ELISA kit (Crystal Chem, Elk Grove Village, IL, USA). The estimated glomerular filtration rate (eGFR) was calculated using the equation as follows:

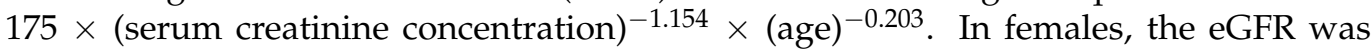
multiplied by 0.742 . Homeostatic model assessment for insulin resistance (HOMA-IR) was calculated using the equation as follows: fasting serum insulin $(\mu \mathrm{U} / \mathrm{mL}) \times$ fasting glucose $(\mathrm{mmol} / \mathrm{L}) / 22.5$.

\subsection{Definition of Osteoarthritis, Obesity, and Metabolic Syndrome}

An arthritis diagnosis was investigated for a diagnosis of OA or rheumatoid arthritis by a physician. They provided the dates when OA or rheumatoid arthritis was diagnosed by a physician or had the diseases when they enrolled in the study. In the present study, the participants with rheumatoid arthritis were excluded, and the remaining participants were categorized into OA and no osteoarthritis (non-OA) groups. Obesity for Asians is defined as $\geq 25 \mathrm{~kg} / \mathrm{m}^{2}$ [13]. Metabolic syndrome was defined according to the 2005 revised National Cholesterol Education Program-Adult Treatment Panel III criteria for Asia, as described previously $[14,15]$.

\subsection{Assessment of Foods and Nutrient Intake, and Diet Patterns}

The semi-quantitative food frequency questionnaire (SQFFQ) designed for Korean diet patterns was assessed for the long-term food intake of the 5430 participants in the AnsanAnsung studies. The validity and reproducibility of this SQFFQ were evaluated using the four three-day food records for four seasons, similar to the 12-day food records in previous studies in the Korean population [16,17]. The adjusted correlation coefficients between the SQFFQ and 12-day food records ranged from 0.23 and 0.64 in various food intakes, suggesting that the validation and reproducibility of this SQFFQ were acceptable [16,17]. The SQFFQ requested the participant's average frequency and consumption of 106 food items during the last year. The intake of food frequencies was categorized into never or seldom, once a month, two to three times a month, one to two times a week, three to four times a week, five to six times a week, once a day, twice a day, and three times or more every day. The amount of food intake at once was checked for "more", "equal", or "less" based on a regular and defined portion size. The food intake per day in each food category was calculated by multiplying the midpoint of the reported frequency category for each food 
item by the food amount at once. The daily intake was calculated based on the midpoint of the reported frequency category for each food item. For example, when the frequency of one food item was noted at five-six/week, it was calculated to be 5.5/7 or 0.79 times/day.

The daily nutrient intake was calculated from the food intake from the SQFFQ. The energy and nutrients, such as protein, carbohydrates, fat, fiber, total vitamin A, vitamin $\mathrm{C}, \mathrm{Na}, \mathrm{Ca}$, and $\mathrm{K}$, were calculated by converting the foods to nutrients using the Can-Pro 2.0 nutrient intake assessment software developed by the Korean Nutrition Society (Seoul, Korea). The daily Estimated Energy Requirement (EER) and recommended nutrient intake were based on the Korean Dietary Reference Intake (DRI) according to age and gender [14].

The 106 food items were divided into 29 food groups used as independent variables during factor analysis to determine the dietary patterns by PCA. The number of factors in the principal component analysis (PCA) were assigned using the eigenvalues $>1.5$, and the orthogonal rotation procedure was applied [15]. Three distinct dietary factors were selected, and dietary factor-loading values of $\geq 0.40$ were considered significant contributions to the dietary patterns. The dietary patterns represented Western-style, plant-based, and rice-main diets (Table S1). The Western-style diet was rich in bread, cookies, mushrooms, fish, crabs, meats, processed meats, beverages, and fast food, while the plant-based diet was high in beans, potatoes, kimchi, vegetables, pickles, seaweed, and fruit. The rice-main diet was high in rice only.

\subsection{Genotyping and Quality Control}

The participants' genotyping and quality-control processes were conducted in the Center for Genome Science, Korea National Institute of Health, as described previously [9]. Briefly, deoxyribonucleic acid (DNA) samples of the participants were isolated from the peripheral blood of the participants and genotyped using the Korean Chip included SNPs involved in the prevalent diseases for Koreans (Affymetrix, Santa Clara, CA, USA). The genotyping accuracy was examined using Bayesian Robust Linear Modeling with the Mahalanobis distance genotyping algorithm [18]. Samples with low genotyping accuracies of $<98 \%$, high missing genotype call rates $(\geq 4 \%)$, high heterozygosity $(>30 \%)$, or gender biases were excluded. The Institutional Review Board of the Korean National Institute of Health (KBP-2019-055) and Hoseo University (1041231-150811-HR-034-01) approved the KoGES and the present studies. All participants provided written informed consent, and they provided the quality-controlled genotype data.

\subsection{Expression Quantitative Trait Locus (eQTL) Analysis}

eQTL analysis is a direct approach to identify the candidate gene expression of risk loci. The allele variants are associated with the corresponding gene expression, and eQTL analysis identified the candidate susceptible genes in various diseases. Gene expression of the genetic variants related to OA risk was identified by eQTL analysis in the GenotypeTissue Expression (GTE) × eQTL calculator (https:/ / gtexportal.org/home/tetyourown (accessed on 15 July 2021)). Because the gene expressions in the articular cartilage were not provided in the GTE $\times$ eQTL calculator, they were calculated indirectly in the skeletal muscle and adipose tissues, as they are involved in OA risk.

\subsection{The Best Model with SNP-SNP Interactions to Influence Osteoarthritis Risk}

Figure 1 presents the selection of genetic variants for the best model for genetic variant-genetic variant interactions. The genetic variants explored the association with OA risk by genome-wide association study (GWAS) of the OA and non-OA groups in the Ansan-Ansung cohort. From the GWAS associated with OA risk, 844 genetic variants were selected at $p<5 \times 10^{-5}$. The gene names of the 844 SNPs were identified using g:Profiler (https:/ / biit.cs.ut.ee/gprofiler/snpense (accessed on 20 June 2021)), and 53 genes were identified. The 25 obesity-related genes (443 SNPs) in the literature were selected using the Human Genome Epidemiology (HuGE) Navigator (https:/ / phgkb.cdc.gov / PHGKB/ hNHome.action (accessed on 29 July 2021)). The corresponding linkage disequilibrium (LD) 
analyses were carried out on the SNPs of the selected genes in the identical chromosomes using Haploview 4.2 in PLINK. The SNPs with high $\mathrm{D}^{\prime}$ values ( $\mathrm{D}^{\prime}>0.3$ ) were not included in GMDR because they provided the same information on the genetic impact.

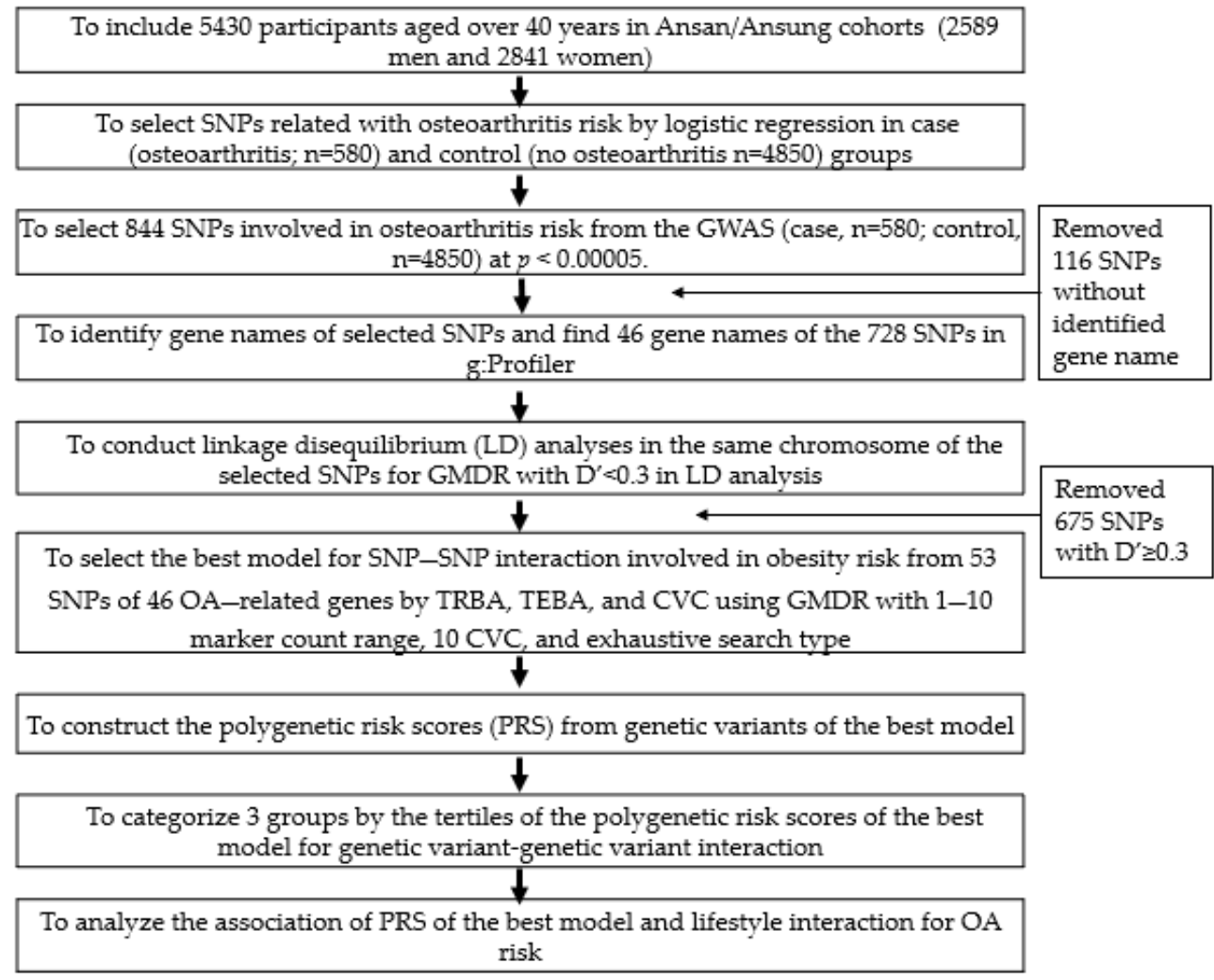

Figure 1. Flow chart for generating polygenetic variants with genetic variant-genetic variant interactions that influence osteoarthritis risk and to explore the interactions between polygenetic risk scores (PRS) and lifestyle.

Of the 46 potential genetic variants in the 25 obesity-related genes, ten SNPs exhibiting a SNP-SNP interaction strongly associated with an obesity risk were selected automatically by the generalized multifactor dimensionality reduction (GMDR) analysis. GMDR provided ten potential models among the different combinations of the 46 genetic variants. The best SNP-SNP interaction model was selected in a sign rank test of trained balanced accuracy (TRBA) and testing balanced accuracy (TEBA) with or without adjusting for the covariates using a GMDR program and a $p$-value threshold of 0.05 [19]. The covariates used were age, gender, residence area, education, income, occupation, energy intake, alcohol consumption, regular exercise, and smoking status. Ten-fold cross-validation was also checked for crossvalidation consistency (CVC) because the sample size was larger than 1000 [19]; 10 out of 10 in CVC met the perfect cross-validation criteria. Using the best model determined by GMDR analysis, the risk allele of each SNP in the selected best model was counted as 1. For example, when people had AA, AG, and GG in one SNP, and the A allele was the risk allele, the genetic score for the SNP was 2, 1, and 0, respectively. The polygenetic-risk scores (PRS) for the best gene-gene-interaction model were assessed by summing the number of the risk alleles (genetic score) from each selected SNP in the best gene-gene-interaction model [20-22]. The PRS in the five and seven SNP models was divided into three categories according to the total number of risk alleles. They were classified as Low-PRS, Middle-PRS, and High-PRS when the number of risk alleles in the PRS was 0-3,4-5, and $\geq 6$ in the five-SNP model and $0-4,5-6$, and $\geq 7$ in the six-SNP model, respectively. 


\subsection{Statistical Analysis}

Statistical analysis was conducted using PLINK version 2.0 (http:/ / pngu.mgh.harvard. edu/ purcell/plink (accessed on 27 May 2021) and SAS version 9.3 (SAS Institute, Cary, NC, USA). Descriptive statistics of the categorical variables (e.g., gender and smoking status) were analyzed using the frequency distributions, and their statistical analysis was evaluated using a Chi-squared test. The descriptive values of the continuous variables were expressed as the means and standard deviations according to the PRS or OA categories. The significance of the differences among the OA or PRS groups was analyzed using a one-way analysis of variance (ANOVA) to adjust the covariates, including age, gender, body mass index, education, income, energy intake (percentage of estimated energy requirement), residence area, daily activity, alcohol intake, and smoking status. Finally, multiple comparisons among the PRS groups were performed using a Tukey's test.

The associations among the PRSs were obtained using the best model, and the obesity risk was examined using multivariate logistic regression analysis with an adjustment for the covariates. The odds ratios (ORs) and 95\% confidence intervals (CI) were calculated as a function of the index reference: Low-PRS. Multivariate logistic regression analysis was conducted using two adjusted models. The covariates of model 1 included gender, age, residence area, education, and income. In contrast, model 2 contained the covariates in model 1 plus smoking status, alcohol consumption, daily energy intake, and regular exercise. The adjusted ORs and 95\% CIs were calculated for obesity risk according to PRS.

The participants were categorized into higher and lower intake groups using the above classification criterion. A multivariate interaction model was used to examine the interactions between the PRS and lifestyle and demographic parameters after adjusting for the covariates for model 2. $p$-values $<0.05$ were considered significant.

\section{Results}

\subsection{Characteristics of the Participants According to the Incidence of $O A$}

OA participants were older than those without OA $(p<0.001)$, and participants aged $\geq 55$ years had a 2.5 -times higher risk than those aged $<55$. In the stratification with the age of the participants, OA incidence was elevated with age, and OA risk was higher by 6.4 times in participants aged $\geq 65$ years than those aged $<45$ years $(p<0.001$; Table 1$)$. The incidence of OA was much lower in men $(19.5 \%)$ than women $(80.5 \%)(p<0.001)$, and women had a 3.2-times higher OA risk than men. The OA participants were diagnosed on average 9.98 years ago, and they still had some pain due to OA (Table 1). The participants with lower education and income had a higher incidence of OA than the others. Education and income were associated with OA risk; higher education and income were inversely associated with OA risk (Table 1).

Height was not significantly different between the OA and control groups. The participants with OA had higher BMI, waist circumference, and fat mass than those without OA (Table 1), but lean body mass did not differ between the OA and non-OA groups. BMI, waist circumference, and fat mass, but not lean body mass, were positively associated with OA risk. Therefore, obesity was associated with OA risk. The serum CRP concentration, an inflammation index, was similar in the OA and non-OA groups (Table 1). The OA group included more participants with metabolic syndrome than the non-OA group, but there was no association between metabolic syndrome and OA risk. The components of metabolic syndrome, including serum glucose, total cholesterol, high-density lipoprotein (HDL), low-density lipoprotein and triglyceride concentrations, systolic blood pressure, and diastolic blood pressure were similar in the OA and non-OA groups. No significant associations of the metabolic syndrome components with OA risk were noted (Table 1). Homeostatic model assessment (HOMA) for insulin resistance (HOMA-IR) and HOMA- $\beta$ cell function (HOMA-B) were similar in the OA and non-OA groups. The eGFR was lower in the OA group than the non-OA group, but it was not significantly associated with OA risk (Table 1). 
Table 1. Characteristics of the participants with osteoarthritis.

\begin{tabular}{|c|c|c|c|}
\hline & Control $(n=4850)$ & Osteoarthritis $(n=580)$ & Adjusted ORs and $95 \% \mathrm{CI}$ \\
\hline Age (<55 yr) & $51.1 \pm 0.10^{1}$ & $54.7 \pm 0.32 * * *$ & $2.501(1.989 \sim 3.144)$ \\
\hline$<45$ & $1428(96.8)$ & $48(3.25)^{* * *}$ & 1 \\
\hline $45-54$ & $1826(92.0)$ & $158(7.96)$ & $2.388(1.663 \sim 3.427)$ \\
\hline $55-64$ & $1176(82.4)$ & $251(17.6)$ & $4.344(2.983 \sim 6.326)$ \\
\hline$\geq 65$ & $420(8.66)$ & $123(22.6)$ & $6.415(4.203 \sim 9.790)$ \\
\hline $\operatorname{Sex}(\mathrm{N}, \%$; males $)$ & $2476(51.1)$ & $113(19.5)^{* * *}$ & $3.198(2.257 \sim 4.531)$ \\
\hline Osteoarthritis duration (yrs) & $0 \pm 0$ & $9.98 \pm 0.39$ & \\
\hline Height $(\mathrm{cm})$ & $160.3 \pm 0.08$ & $160.4 \pm 0.24$ & $1.098(0.805 \sim 1.498)$ \\
\hline $\mathrm{BMI}\left(<25 \mathrm{~kg} / \mathrm{m}^{2}\right)$ & $24.5 \pm 0.05$ & $25.4 \pm 0.14^{* * *}$ & $1.488(1.180 \sim 1.876)$ \\
\hline Waist circumferences (M: $<90 \mathrm{~cm} ; \mathrm{F}:<85 \mathrm{~cm})$ & $82.2 \pm 0.12$ & $84.6 \pm 0.37^{* * *}$ & $1.372(1.106 \sim 1.703)$ \\
\hline Lean body mass (M: $<35 \% ; \mathrm{F}:<28 \%$ ) & $31.1 \pm 0.05$ & $31.5 \pm 0.12$ & $1.148(0.926 \sim 1.424)$ \\
\hline Fat mass $(\mathrm{M}:<25 \% ; \mathrm{F}:<30 \%)$ & $26.5 \pm 0.08$ & $27.7 \pm 0.26^{* * *}$ & $1.246(1.005 \sim 1.545)$ \\
\hline \multicolumn{4}{|l|}{ Education $(\mathrm{N}, \%)$} \\
\hline$\leq$ Middle school & $2421(84.3)$ & $452(15.7) * * *$ & 1 \\
\hline High school & $1687(94.4)$ & $100(5.60)$ & $0.711(0.531 \sim 0.951)$ \\
\hline$\geq$ College & $723(96.8)$ & $24(3.21)$ & $0.605(0.376 \sim 0.974)$ \\
\hline \multicolumn{4}{|l|}{ Income $(\mathrm{N}, \%)$} \\
\hline$\leq \$ 2000$ & $1421(81.8)$ & $316(18.2)^{* * *}$ & 1 \\
\hline$\$ 2000-4000$ & $2398(92.2)$ & $203(7.80)$ & $0.777(0.621 \sim 1.001)$ \\
\hline$>\$ 4000$ & $971(95.3)$ & $48(4.71)$ & $0.690(0.469 \sim 1.015)$ \\
\hline $\operatorname{MetS}(\mathrm{N}, \%)$ & $908(16.7)$ & $173(29.8)^{* * *}$ & $1.051(0.814 \sim 1.356)$ \\
\hline Serum glucose $(<126 \mathrm{mg} / \mathrm{dL})$ & $87.5 \pm 0.30$ & $86.7 \pm 0.93$ & $0.864(0.659 \sim 1.135)$ \\
\hline Serum insulin $(<9.5 \mathrm{IU} / \mathrm{L})$ & $7.48 \pm 0.06$ & $7.63 \pm 0.19$ & $0.936(0.760 \sim 1.154)$ \\
\hline $\operatorname{HbA} 1 \mathrm{c}(<6.5 \%)$ & $5.78 \pm 0.01$ & $5.72 \pm 0.04$ & $0.967(0.717 \sim 1.305)$ \\
\hline HOMA-IR $(<1.95)$ & $1.63 \pm 0.02$ & $1.65 \pm 0.04$ & $0.969(0.772 \sim 1.216)$ \\
\hline HOMA-B $(<160)$ & $149.4 \pm 2.09$ & $150.9 \pm 6.50$ & $1.109(0.905 \sim 1.361)$ \\
\hline Serum total cholesterol $(<230 \mathrm{mg} / \mathrm{dL})$ & $192.6 \pm 0.51$ & $194.4 \pm 1.57$ & $0.995(0.762 \sim 1.300)$ \\
\hline Serum HDL (M: <40, F: <50 mg/dL) & $44.7 \pm 0.14$ & $44.6 \pm 0.43$ & $0.984(0.791 \sim 1.225)$ \\
\hline Serum LDL $(<130 \mathrm{mg} / \mathrm{dL})$ & $115.6 \pm 0.48$ & $118.0 \pm 1.49$ & $1.049(0.760 \sim 1.447)$ \\
\hline Serum Triglyceride $(<150 \mathrm{mg} / \mathrm{dL})$ & $161.7 \pm 1.51$ & $159.1 \pm 4.66$ & $1.008(0.822 \sim 1.235)$ \\
\hline Serum CRP $(<0.5 \mathrm{mg} / \mathrm{dL})$ & $0.22 \pm 0.01$ & $0.21 \pm 0.02$ & $0.841(0.451 \sim 1.571)$ \\
\hline $\mathrm{SBP}(<130 \mathrm{mmHg})$ & $116.6 \pm 0.24$ & $117.0 \pm 0.73$ & $0.908(0.723 \sim 1.140)$ \\
\hline DBP (<90 mmHg) & $75.0 \pm 0.16$ & $75.1 \pm 0.48$ & $1.016(0.760 \sim 1.358)$ \\
\hline eGFR (<70 mL/min) & $85.4 \pm 0.23$ & $83.8 \pm 0.71 *$ & $1.021(0.793 \sim 1.314)$ \\
\hline Serum AST $(<40 \mathrm{U} / \mathrm{L})$ & $29.2 \pm 0.26$ & $28.7 \pm 0.81$ & $0.880(0.586 \sim 1.322)$ \\
\hline Serum ALT $(<35 \mathrm{U} / \mathrm{L})$ & $28.3 \pm 0.45$ & $27.8 \pm 1.38$ & $1.051(0.791 \sim 1.396)$ \\
\hline
\end{tabular}

${ }^{1}$ Adjusted means and 95\% confidence intervals after adjusting for covariates included age, gender, education, income, energy intake (percentage of estimated energy requirement), residence area, daily activity, alcohol consumption, and smoking status. * Significantly different from the control group at $p<0.05$ and ${ }^{* * *}$ at $p<0.001$.

\subsection{Nutrient Intake and Lifestyles in the OA Participants}

The daily energy intake based on the estimated energy intake was similar in the OA and non-OA groups. The macronutrient intake, including carbohydrate, protein, and fat, was similar in the two groups (Table 2). No significant differences in the saturated, monounsaturated, and polyunsaturated fatty acid intake were observed between the OA and non-OA groups. Other nutrient intake, including cholesterol, vitamin C, and fiber intake, did not affect OA risk (Table 2). Unlike the nutrient intake, dietary patterns categorized by principal component analysis (PCA) from the semi-quantitative food frequency questionnaire (SQFFQ) showed a significant difference between the OA and non-OA groups. The dietary patterns were divided into a Western-style diet, plant-based diet, and rice-main diet. Consistent with a high protein diet, the Western-style diet was inversely associated with OA risk (Table 2). However, OA incidence did not differ in a low and high plant-based diet, and rice-main diet groups; and the diet patterns were not significantly associated with OA risk (Table 2). OA incidence was lower in the smoker group than the non-smoker group, while higher in the exercise group than the non-exercise group. However, alcohol 
consumption, smoking, and regular exercise were not significantly associated with OA risk (Table 2).

Table 2. Lifestyle including nutrient intake and association with obesity in the participants according to genders and obese status.

\begin{tabular}{|c|c|c|c|}
\hline & $\begin{array}{c}\text { Control } \\
(n=4850)\end{array}$ & Osteoarthritis $(n=580)$ & Adjusted ORs and $95 \%$ CI \\
\hline Energy $(<\text { EER\% })^{1}$ & $102.7 \pm 0.53^{2}$ & $105.4 \pm 1.65$ & $1.175(0.966 \sim 1.428)^{3}$ \\
\hline Carbohydrates (<70 En\%) & $70.8 \pm 0.09$ & $70.8 \pm 0.29$ & $1.055(0.836 \sim 1.332)$ \\
\hline Fiber $(<20 \mathrm{~g} / \mathrm{d})$ & $21.3 \pm 0.18$ & $21.7 \pm 0.56$ & $0.966(0.771 \sim 1.211)$ \\
\hline Protein $(<13 \mathrm{En} \%)$ & $13.5 \pm 0.03$ & $13.6 \pm 0.10$ & $0.957(0.775 \sim 1.183)$ \\
\hline Fat $(<15$ En\%) & $14.6 \pm 0.07$ & $14.5 \pm 0.22$ & $0.975(0.772 \sim 1.230)$ \\
\hline Saturated fat $(<5.7 \mathrm{En} \%)$ & $4.2 \pm 0.4$ & $4.3 \pm 1.1$ & $1.045(0.798 \sim 1.368)$ \\
\hline Monounsaturated fat (<7.0 En\%) & $5.4 \pm 0.4$ & $5.5 \pm 1.2$ & $0.979(0.737 \sim 1.299)$ \\
\hline Polyunsaturated fat (<3.5 En\%) & $2.6 \pm 0.2$ & $2.6 \pm 0.5$ & $0.880(0.640 \sim 1.210)$ \\
\hline Cholesterol $(<250 \mathrm{mg} / \mathrm{d})$ & $177 \pm 1.57$ & $179 \pm 4.85$ & $0.936(0.705 \sim 1.243)$ \\
\hline $\operatorname{Vitamin} C(<100 \mathrm{mg} / \mathrm{d})$ & $128 \pm 1.13$ & $126 \pm 3.50$ & $0.932(0.701 \sim 1.239)$ \\
\hline Plant-based diet (<70th percentile) & $1588(32.7)^{4}$ & $211(36.4)$ & $1.180(0.944 \sim 1.476)$ \\
\hline Western-style diet ( $<70$ th percentile) & $1691(34.9)$ & $113(19.5)^{* * *}$ & $0.726(0.550 \sim 0.957)$ \\
\hline Rice-main diet (<70th percentile) & $1623(33.4)$ & $173(29.8)$ & $1.081(0.866 \sim 1.349)$ \\
\hline Flavonoids (<70th percentile) & $64.2 \pm 0.82$ & $61.8 \pm 2.56$ & $0.965(0.758 \sim 1.228)$ \\
\hline Alcohol drinking $(<20 \mathrm{~g} / \mathrm{d})$ & $9.85 \pm 0.29$ & $10.2 \pm 0.90$ & $1.061(0.702 \sim 1.602)$ \\
\hline Smoking (current smokers) & $1220(25.6)$ & $58(10.2) * * *$ & $0.802(0.501 \sim 1.285)$ \\
\hline Regular exercise (<150 min/week) & $1379(29.2)$ & $224(40.5) * * *$ & $1.212(0.955 \sim 1.538)$ \\
\hline
\end{tabular}

${ }^{1}$ The cutoff points for logistic regression. ${ }^{2}$ The values represent adjusted means \pm standard errors. ${ }^{3}$ Adjusted odds ratio (ORs) and 95\% confidence intervals after adjusting for covariates included age, gender, education, income, energy intake (percentage of estimated energy requirement), residence area, daily activity, alcohol consumption, and smoking status. ${ }^{4}$ The number of the subjects (percentage of each group). ${ }^{* *}$ Significantly different from the control group at ${ }^{* * *} p<0.001$.

\subsection{Genetic Variants Related to OA Risk and the Best Model with Genetic Variant-Genetic Variant Interaction}

After GWAS for OA risk in the Ansan-Ansung cohort and removing some SNPs not meeting the inclusion criteria (Figure 1), only two genetic variants, including androgen induced 1 (AIG1)_rs6570550 and cytochrome C oxidase assembly factor heme A (COX10)_rs62054459, satisfied the statistical significance of the Bonferroni correction. Fiftythree SNPs containing rs6570550 and rs62054459 were included for determining the best genetic variant-genetic variant interaction model with these two SNPs. Ten SNPs were selected because they showed genetic variant-genetic variant interactions, and 5-10 SNPs in the models met the best model criteria: $p$-value $<0.05$ for the sign test of TRBA and TEBA, and CVC $=9$ or 10 in GMDR analysis. The characteristics of 10 SNPs are presented in Table 3 . The five-SNP model for OA risk included AIG1_rs6570550, COX10_rs62054459, discs large MAGUK scaffold protein 2 (DLG2)_rs148643344, plexin A4 (PLXNA4)_rs1472529430, and SRY-box transcription factor 5 (SOX5)_rs73283615 (Table S2). The six-SNP model contained the genetic variants in the five-SNP model plus interleukin 12A (IL12A)_rs1491318751 (Table S2).

ORs and 95\% CI for the PRS of the five- and six-SNP best models of SNP-SNP interaction were 3.89 (2.723-5.548) and 4.42 (3.211-6.09), respectively, in model 2 adjusted for age, gender, BMI, OA duration, the status of smoking and drinking, levels of income and education, job, income, physical activity, energy intake, percentage intake of carbohydrate and fat, and arthritis medication (Table 4). Each SNP selected in the best model was not significantly associated with rheumatoid arthritis, but the PRS of the five-SNP and sixSNP models was significantly associated with the rheumatoid arthritis risk (ORs $=2.041$, $95 \% \mathrm{CI}=1.258-3.311$ for the five-SNP and $\mathrm{ORs}=1.855,95 \% \mathrm{CI}=1.202-2.862)$ for the six-SNP model. On the other hand, metabolic syndrome and HOMA-IR, which have 
potentially related etiologies, were not significantly associated with the PRS of the five-SNP and six-SNP models (Table 4).

Table 3. The characteristics of the ten genetic variants of genes related to osteoarthritis in adults using the generalized multifactor dimensionality reduction analysis.

\begin{tabular}{|c|c|c|c|c|c|c|c|c|c|c|c|}
\hline $\mathrm{CHR}^{1}$ & $\mathrm{SNP}^{2}$ & Location & $\mathrm{Mi}^{3}$ & $\mathrm{OR}^{4}$ & L95 ${ }^{5}$ & U95 ${ }^{6}$ & $\begin{array}{c}p \text {-Value for } \\
\text { OR }^{7}\end{array}$ & Genes & Feature & MAF $^{8}$ & HWE $^{9}$ \\
\hline 3 & rs149045369 & 129206303 & $\mathrm{~T}$ & 0.324 & 0.1925 & 0.5449 & $2.16 \times 10^{-5}$ & IFT122 & transcript & 0.0526 & 0.786 \\
\hline 3 & rs1491318751 & 159765533 & G & 1.639 & 1.3 & 2.067 & $2.94 \times 10^{-5}$ & IL12A & intron & 0.0974 & 1 \\
\hline 6 & rs6913416 & 157454046 & $\mathrm{C}$ & 2.559 & 1.659 & 3.947 & $2.14 \times 10^{-5}$ & $A R I D 1 B$ & intron & 0.0194 & 1 \\
\hline 6 & rs6570550 & 143480314 & A & 1.572 & 1.338 & 1.847 & $3.73 \times 10^{-8}$ & AIG1 & intron & 0.3139 & 0.117 \\
\hline 7 & rs1472529430 & 132018047 & $\mathrm{~T}$ & 0.602 & 0.4785 & 0.7564 & $1.37 \times 10^{-5}$ & PLXNA4 & intron & 0.1802 & 1 \\
\hline 11 & rs148643344 & 85026573 & G & 1.765 & 1.379 & 2.258 & $6.29 \times 10^{-6}$ & DLG2 & intron & 0.0771 & 0.128 \\
\hline 12 & rs73283618 & 24112286 & C & 1.419 & 1.214 & 1.658 & $1.05 \times 10^{-5}$ & SOX5 & intron & 0.3767 & 0.752 \\
\hline 17 & rs62054459 & 13672047 & $\mathrm{~T}$ & 0.567 & 0.4626 & 0.6939 & $3.96 \times 10^{-8}$ & COX10 & intron & 0.232 & 0.733 \\
\hline 17 & rs138377463 & 43069398 & $\mathrm{~A}$ & 1.927 & 1.401 & 2.65 & $5.43 \times 10^{-5}$ & NMT1 & intron & 0.0434 & 0.518 \\
\hline 20 & rs141079635 & 41491626 & C & 2.077 & 1.482 & 2.912 & $2.18 \times 10^{-5}$ & PTPRT & intron & 0.0382 & 0.357 \\
\hline
\end{tabular}

${ }^{1}$ Chromosome; ${ }^{2}$ Single nucleotide polymorphism; ${ }^{3}$ Minor allele; ${ }^{4}$ Odds ratio; ${ }^{5}$ Lower and ${ }^{6}$ upper ends of $95 \%$ confidence interval; ${ }^{7} p$-value for OR after adjusting for age, gender, residence area, survey year, body mass index, daily energy intake, levels of education and income; ${ }^{8}$ Minor allele frequency; ${ }^{9}$ Hardy-Weinberg equilibrium.

Table 4. Adjusted odds ratios for osteoarthritis according to the polygenetic risk scores of the best model (PRS) for gene-gene interaction after covariate adjustments.

\begin{tabular}{|c|c|c|c|c|c|}
\hline \multirow[b]{2}{*}{ For 5-SNP Model } & \multicolumn{3}{|c|}{ Model 1} & \multicolumn{2}{|c|}{ Model 2} \\
\hline & $\begin{array}{c}\text { Low-PRS } \\
(n=2373)^{1}\end{array}$ & $\begin{array}{l}\text { Medium-PRS } \\
\quad(n=1583)\end{array}$ & $\begin{array}{l}\text { High-PRS } \\
(n=1474)\end{array}$ & $\begin{array}{l}\text { Medium-PRS } \\
\quad(n=1583)\end{array}$ & $\begin{array}{c}\text { High-PRS } \\
(n=1474)\end{array}$ \\
\hline Osteoarthritis & 1 & $\begin{array}{c}2.332^{2} \\
(1.853 \sim 2.935)^{2}\end{array}$ & $\begin{array}{c}3.708 \\
(2.617 \sim 5.254)\end{array}$ & $\begin{array}{c}2.381 \\
(1.876 \sim 3.021)\end{array}$ & $\begin{array}{c}3.887 \\
(2.723 \sim 5.548)\end{array}$ \\
\hline $\begin{array}{l}\text { Rheumatoid } \\
\text { arthritis }\end{array}$ & 1 & $\begin{array}{c}1.309 \\
(0.978 \sim 1.752)\end{array}$ & $\begin{array}{c}1.807 \\
(1.128 \sim 2.894)\end{array}$ & $\begin{array}{c}1.316 \\
(0.961 \sim 1.801)\end{array}$ & $\begin{array}{c}2.041 \\
(1.258 \sim 3.311)\end{array}$ \\
\hline $\begin{array}{l}\text { Metabolic } \\
\text { syndrome }\end{array}$ & 1 & $\begin{array}{c}0.908 \\
(0.773 \sim 1.067)\end{array}$ & $\begin{array}{c}0.910 \\
(0.679 \sim 1.218)\end{array}$ & $\begin{array}{c}0.886 \\
(0.744 \sim 1.055)\end{array}$ & $\begin{array}{c}0.855 \\
(0.624 \sim 1.169)\end{array}$ \\
\hline HOMA-IR & 1 & $\begin{array}{c}1.063 \\
(0.945 \sim 1.196)\end{array}$ & $\begin{array}{c}1.028 \\
(0.829 \sim 1.274)\end{array}$ & $\begin{array}{c}1.080 \\
(0.952 \sim 1.225)\end{array}$ & $\begin{array}{c}1.030 \\
(0.821 \sim 1.293)\end{array}$ \\
\hline For 6-SNP model & $\begin{array}{c}\text { Low-PRS } \\
(n=2100)^{3}\end{array}$ & $\begin{array}{l}\text { Medium PRS } \\
\quad(n=2658)\end{array}$ & $\begin{array}{c}\text { High PRS } \\
(n=672)\end{array}$ & $\begin{array}{c}\text { Medium PRS } \\
\quad(n=2658)\end{array}$ & $\begin{array}{l}\text { High PRS } \\
(n=672)\end{array}$ \\
\hline Osteoarthritis & 1 & $\begin{array}{c}2.062 \\
(1.619 \sim 2.626)\end{array}$ & $\begin{array}{c}4.165 \\
(3.051 \sim 5.687)\end{array}$ & $\begin{array}{c}2.087 \\
(1.624 \sim 2.681)\end{array}$ & $\begin{array}{c}4.422 \\
(3.211 \sim 6.09)\end{array}$ \\
\hline $\begin{array}{l}\text { Rheumatoid } \\
\text { arthritis }\end{array}$ & 1 & $\begin{array}{c}1.173 \\
(0.867 \sim 1.585)\end{array}$ & $\begin{array}{c}1.735 \\
(1.146 \sim 2.626)\end{array}$ & $\begin{array}{c}1.181 \\
(0.857 \sim 1.627)\end{array}$ & $\begin{array}{c}1.855 \\
(1.202 \sim 2.862)\end{array}$ \\
\hline $\begin{array}{l}\text { Metabolic } \\
\text { syndrome }\end{array}$ & 1 & $\begin{array}{c}0.912 \\
(0.772 \sim 1.078)\end{array}$ & $\begin{array}{c}0.841 \\
(0.656 \sim 1.078) \\
\end{array}$ & $\begin{array}{c}0.879 \\
(0.732 \sim 1.056)\end{array}$ & $\begin{array}{c}0.802 \\
(0.611 \sim 1.053)\end{array}$ \\
\hline HOMA-IR & 1 & $\begin{array}{c}1.027 \\
(0.910 \sim 1.160)\end{array}$ & $\begin{array}{c}0.974 \\
(0.810 \sim 1.170)\end{array}$ & $\begin{array}{c}1.052 \\
(0.925 \sim 1.198)\end{array}$ & $\begin{array}{c}0.977 \\
(0.803 \sim 1.190)\end{array}$ \\
\hline
\end{tabular}

${ }^{1}$ Number of participants in each PRS category of the 5-SNP model: Low-PRS ( $\left.<4\right)$, Medium-PRS (4-6), High-PRS (>6) for 5-SNP model. ${ }^{2}$ Values were expressed as odds ratio and $95 \%$ confidence intervals after adjusting for covariates including age, gender, education, income, and residence areas for model 1, parameters of model 1 plus energy intake (percentage of estimated energy requirement), physical activity, alcohol intake, and smoking status for model 2. ${ }^{3}$ Number of participants in each PRS category of the 6-SNP model: Low-PRS $(<5)$, Medium-PRS (5-7), High-PRS (>7) for 6-SNP model.

\section{4. eQTL Analysis in Skeletal Muscle and Adipose Tissue}

Among the 10 genetic variants related to OA risk, COX10_rs62054459 had a significant eQTL association. The minor allele of COX10_rs62054459 had a higher expression of COX10 in subcutaneous and visceral adipose tissue but not the skeletal muscle, than its major allele 
(Figure 2). AIG1 showed a tendency to an eQTL association, but it was not significantly associated (Figure 2).

A

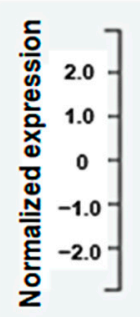

D

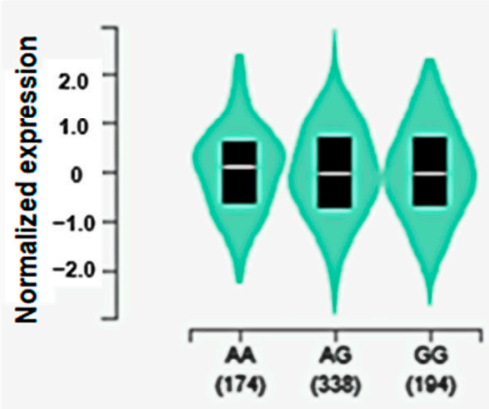

B

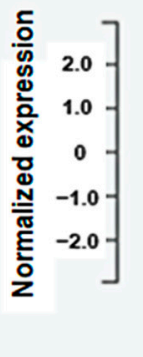

E

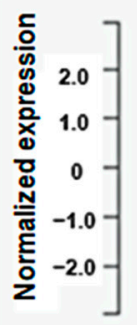

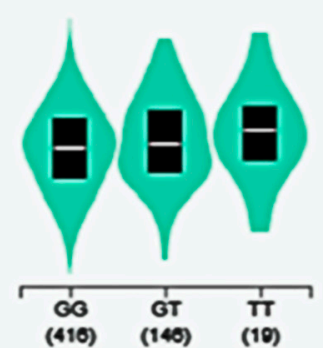

(410) (140) (19)

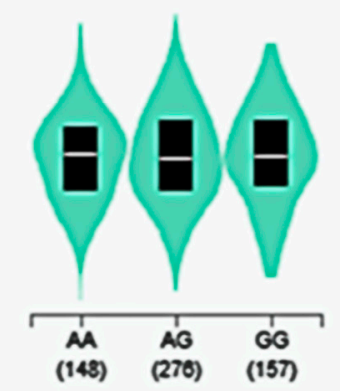

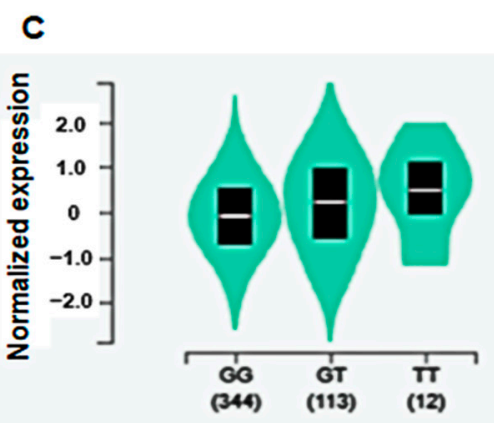

$\mathbf{F}$
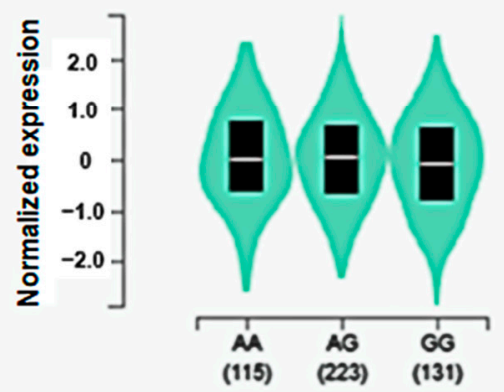

Figure 2. Gene expression of COX10_rs62054459 and AIG1_rs6570550 in the skeletal muscle, subcutaneous and visceral adipose tissue by eQTL analysis. Gene expression of the genetic variants to influence OA risk was identified by eQTL analysis in the Genotype-Tissue Expression (GTE) $\times$ eQTL calculator. (A). COX10_rs62054459 in the skeletal muscle ( $p=0.47)$; (B). COX10_rs62054459 in subcutaneous adipose tissue $(p=0.015)$; (C). COX10_rs62054459 in visceral adipose tissue $(p=0.014)$; (D). AIG1_rs6570550 in skeletal muscle ( $p=1.0)$; (E). AIG1_rs6570550 in subcutaneous adipose tissue $(p=0.41) ;(\mathbf{F})$. AIG1_rs6570550 in visceral adipose tissue $(p=0.062)$.

\subsection{Interaction of PRS and Nutrient Intake in OA Risk}

Although the participants were diagnosed with OA approximately $9.98 \pm 0.39$ years before enrolling in the Ansan-Ansung cohort study, they had OA symptoms when they enrolled in the Ansan-Ansung cohort study. The dietary intake involved OA symptoms by interacting with the PRS. The PRS from the five-SNP and six-SNP models showed a similar interaction, and the interaction results of the PRS were provided from the five-SNP model. The energy intake interacted with the PRS to influence OA risk ( $p=0.0048)$ : in high energy intake, OA risk was much higher in the high-PRS group than in the low-PRS group (Table 5 and Figure 3A). Among energy intake, carbohydrate intake did not interact with PRS to influence OA risk (Table 5), and OA incidence increased with PRS in both highand low-CHO groups (Figure 3B). The protein and fat intakes showed an interaction with the PRS to affect OA risk; in the low protein $(p=0.0367)$ and fat intake $(p=0.0420)$, the participants with a high-PRS had a much higher OA incidence than those with a low-PRS (Table 5 and Figure 3C,D). As with the high protein diet, WSD had an interaction with PRS $(p=0.0304$; Table 5), and the increase of OA incidence with PRS was lower in a high WSD than in a low WSD (Figure 3E). However, a plant-based and rice-main diet did not interact with the PRS to influence OA risk (Table 5). Alcohol intake also interacted with the PRS for OA risk: OA incidence was much higher in the participants with high-PRS than low-PRS in the participants with a low alcohol intake, but not those with a high alcohol intake (Table 5 and Figure 3F). Regular exercise and smoking status did not interact with the PRS, although OA incidence was positively associated with PRS in both low- and high-exercise groups and smoking status (Table 5). 

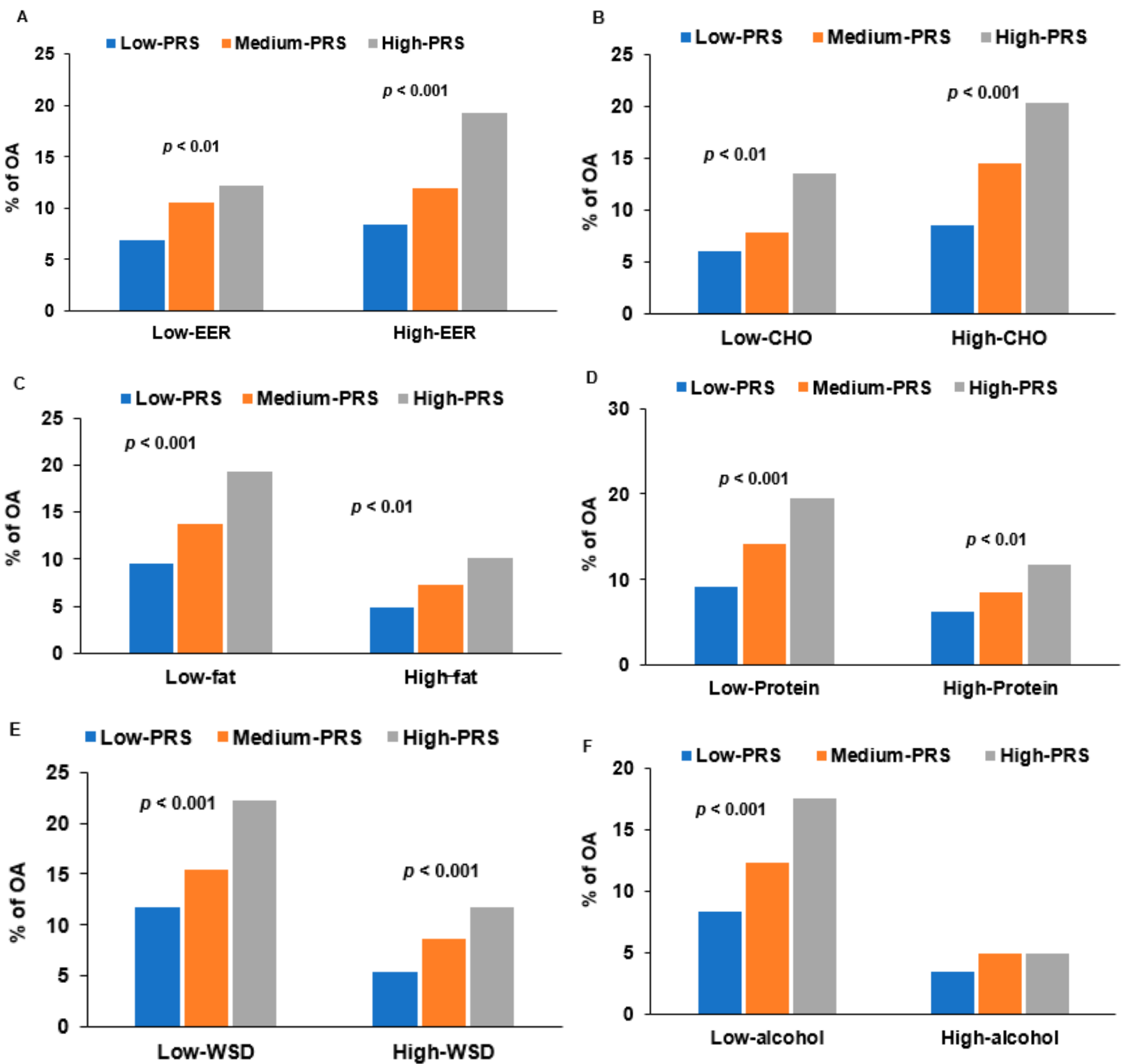

Figure 3. Interaction of nutrient intake with low, medium, or high polygenetic risk scores (PRS) from the 5-SNP model to influence osteoarthritis (OA) risk. (A). OA incidence (\%) of the participants categorized by daily energy intake (cutoff value: estimated energy requirement (EER) according to age and genders). (B). OA incidence (\%) of the participants categorized by carbohydrate $(\mathrm{CHO})$ intake (cutoff value: 70 energy percent). (C). OA incidence (\%) of the participants categorized by fat intake (cutoff value: 15 energy percent). (D). OA incidence (\%) of the participants categorized by protein intake (cutoff value: 13 energy percent). (E). OA incidence (\%) of the participants categorized by Western-style diet intake (cutoff value: 70th percentile). (F). OA incidence (\%) of the participants by alcohol intake (cutoff value: $20 \mathrm{~g}$ alcohol/day). Covariates included age, gender, body mass index, OA duration, the status of smoking and drinking, levels of income and education, job, income, physical activity, energy intake, percent intake for carbohydrate and fat, and arthritis medication. 
Table 5. Adjusted odds ratios for osteoarthritis risk by polygenetic risk scores of the 5-SNP best model (PRS) for gene-gene interaction after covariate adjustments according to lifestyle patterns.

\begin{tabular}{|c|c|c|c|c|}
\hline & $\begin{array}{l}\text { Low-PRS } \\
(n=2373)^{1}\end{array}$ & $\begin{array}{l}\text { Medium-PRS } \\
\quad(n=1583)\end{array}$ & $\begin{array}{l}\text { High-PRS } \\
(n=1474)\end{array}$ & $\begin{array}{c}\text { PRS-Nutrient Interaction } \\
p \text {-Value } \\
{ }^{3}\end{array}$ \\
\hline Low energy & \multirow{2}{*}{1} & $1.797(1.290 \sim 2.503)^{2}$ & $3.010(1.937 \sim 4.678)$ & \multirow{2}{*}{0.0048} \\
\hline High energy 4 & & $1.934(1.400 \sim 2.673)$ & $5.137(3.396 \sim 7.770)$ & \\
\hline Low carbohydrate & \multirow[b]{2}{*}{1} & 1.631 (1.091 2.439) & $3.567(2.200 \sim 5.785)$ & \multirow[b]{2}{*}{0.0864} \\
\hline High carbohydrate 5 & & 1.905 (1.450 2.502) & $3.878(2.677 \sim 5.616)$ & \\
\hline Low protein & \multirow[b]{2}{*}{1} & $1.979(1.462 \sim 2.678)$ & $4.201(2.794 \sim 6.317)$ & \multirow[b]{2}{*}{0.0367} \\
\hline High protein 6 & & $1.619(1.154 \sim 2.272)$ & $3.385(2.217 \sim 5.168)$ & \\
\hline Low fat & \multirow{2}{*}{1} & $1.751(1.343 \sim 2.283)$ & $3.838(2.682 \sim 5.494)$ & \multirow{2}{*}{0.0420} \\
\hline Moderate fat ${ }^{7}$ & & $2.019(1.315 \sim 3.100)$ & $3.641(2.168 \sim 6.117)$ & \\
\hline Low alcohol & \multirow[b]{2}{*}{1} & $1.865(1.474 \sim 2.360)$ & $4.011(2.948 \sim 5.459)$ & \multirow[b]{2}{*}{0.0207} \\
\hline High alcohol ${ }^{8}$ & & $1.333(0.603 \sim 2.948)$ & $2.403(0.907 \sim 6.366)$ & \\
\hline Low WSD & \multirow{2}{*}{1} & $1.742(1.249 \sim 2.430)$ & $4.127(2.784 \sim 6.116)$ & \multirow{2}{*}{0.0304} \\
\hline High WSD ${ }^{9}$ & & $1.949(1.413 \sim 2.690)$ & $3.776(2.361 \sim 6.039)$ & \\
\hline Low PBD & \multirow[b]{2}{*}{1} & $2.244(1.478 \sim 3.409)$ & $4.103(2.362 \sim 7.128)$ & \multirow[b]{2}{*}{0.5343} \\
\hline High PBD ${ }^{10}$ & & $1.729(1.309 \sim 2.282)$ & $3.855(2.698 \sim 5.508)$ & \\
\hline Low RMD & \multirow[b]{2}{*}{1} & $1.555(1.070 \sim 2.261)$ & $3.817(2.350 \sim 6.198)$ & \multirow[b]{2}{*}{0.0591} \\
\hline High RMD 11 & & 2.079 (1.548 2.793) & $4.015(2.741 \sim 5.881)$ & \\
\hline Low exercise & \multirow[b]{2}{*}{1} & $2.163(1.601 \sim 2.922)$ & $4.633(3.167 \sim 6.778)$ & \multirow[b]{2}{*}{0.1367} \\
\hline High exercise $^{12}$ & & $1.484(1.051 \sim 2.096)$ & $2.904(1.820 \sim 4.635)$ & \\
\hline Non-smoker & \multirow[b]{2}{*}{1} & $1.882(1.478 \sim 2.395)$ & $4.583(3.349 \sim 6.270)$ & \multirow{2}{*}{0.1207} \\
\hline Smoker + former smoker & & $1.721(1.363 \sim 2.174)$ & $3.719(2.735 \sim 5.056)$ & \\
\hline
\end{tabular}

${ }^{1}$ PRS was divided into three categories $(0-3,4-6$, and $>6$ ) by three groups as the low, medium, and high groups of the 5-SNP best model of GMDR. Low-PRS was the reference. ${ }^{2}$ Values were expressed as odds ratio and $95 \%$ confidence intervals. ${ }^{3}$ Multivariate regression models include the main effects, interaction terms of gene and main effects (energy and nutrient intake), and potential confounders including sex, age, BMI, the status of smoking and drinking, levels of income and education, job, physical activity, hypertension, energy, milk, percent intake of carbohydrate and fat, and medication for arthritis and dermatitis. The cutoff points of the parameters were defined as: ${ }^{4}<$ estimated energy intake, ${ }^{5}<70 \%$ carbohydrate, ${ }^{6}<13 \%$ protein, ${ }^{7}<15 \%$ fat, ${ }^{8}<20 \mathrm{~g} /$ day alcohol, 9-11 Western-style diet (WSD), plant-based diet (PBD), and rice-main diet (RMD), respectively, and ${ }^{12} 150 \mathrm{~min}$ moderate exercise per week.

\section{Discussion}

$\mathrm{OA}$ is a complex polygenetic disease involving genetic and environmental factors that influence OA risk. The incidence of OA is increasing worldwide as the elderly population increases; its increase is significant in older Asian women. Arthritis is a broad name for joint pain and inflammation, but the causes and etiologies are different. OA develops with wear and tear in the articular cartilage of the joints by repeated stress with aging. The symptoms are similar in all cases of arthritis, but the etiology is different. The present study determined the genetic factors for OA risk in the Ansan-Ansung cohort because this cohort included the incidence of arthritis divided into OA and rheumatoid arthritis. On the other hand, the city hospital-based cohort, the larger cohort in KoGES, included arthritis incidence, but it was not separated into OA and rheumatoid arthritis. The present study is novel because it explores the polygenetic impact on $\mathrm{OA}$ and its interaction with nutrient intake in the Ansan-Ansung cohort.

OA develops slowly with age and is accompanied by pain, stiffness, loss of flexibility, grating sensation, bone spurs, and swelling. The disease is involved in the gradual degradation of the extracellular matrix in cartilage and bone. Cartilage is composed mainly of collagen type 2 with aggrecan and proteoglycans. The environmental risk factors for OA have been well established, including older age, women, obesity, repeated stress on the joints, joint injuries, bone deformities. Genetic factors affecting $\mathrm{OA}$ development have been reported, but they remain unclear. A collagen type 2 gene mutation is involved directly in OA incidence in a case report [23]. Cartilage is maintained by its synthesis and degradation, and genetic factors are related. Its degradation is involved in extracellular matrix (ECM) degradation enzymes, such as matrix metalloproteinase (MMP)-1, MMP-3, and MMP-13, 
in chondrocytes and ECM synthesis by the SOX9 expression [24]. However, their genetic variants were not selected for OA risk in the present study.

Obesity is positively associated with OA risk. It is not just due to the heavy load on the knee due to high BMI, but due to increased inflammation by increased adipose tissue $[2,3]$. The present study demonstrated that fat mass was positively associated with OA risk but not lean body mass. OA is involved in inflammation stimuli in synovium inflammation and cartilage degradation through the catabolic activities of chondrocytes [25]. Inflammatory cytokines, including IL-1, IL-4, IL-6, IL-17, IL-18, and tumor necrosis factor (TNF)- $\beta$, stimulate matrix degradation [26]. IL-12 regulates the balance between Th1 and Th2 cells and enhances cytotoxic T cell-mediated lysis and natural killer (NK) cell activity. The IL-12 mRNA and protein levels are higher in rheumatoid arthritis and OA joints than in normal adults $[25,27]$. In the present study, IL12A_rs1491318751 was significantly and positively associated with OA. It is also related to collagen synthesis in the cartilage, while SOX5 is involved in enhanced chondrogenic differentiation and chondrogenesis, and SOX5 suppression, with miR-194 up-regulation, decreases chondrogenesis [28]. In the present study, a person with the minor SOX5_rs73283615 allele was positively associated with OA risk. Therefore, the genetic impact might be related to inflammation and chondrogenic differentiation in OA in Korean adults. Furthermore, the neuronal network contributes to OA-induced pain to exacerbate OA symptoms. In the present study, genetic variants of SOX5, PLXNA4, and DLG2 involved in neuronal networks were involved in OA risk. Therefore, OA risk is associated with synovium inflammation, cartilage degradation, inflammation, and the neuronal network.

COX10_rs62054459 and AIG1_rs6570550 met the statistical significance of the Bonferroni correction $\left(p<5 \times 10^{-8}\right)$, and were included in the best model for OA genetic risk in the present study. People with the AIG1_rs6570550 minor allele were positively associated with OA risk, while those with COX10_ rs62054459 minor alleles were inversely associated with it. COX10 is the terminal component in the mitochondria respiratory chain reaction for the electron transfer from reduced cytochrome $\mathrm{C}$ to oxygen. COX10 knockout mice began to develop progressive myopathy at three months of age, and the myopathy was worsened by aging, particularly in female mice [29]. On the other hand, no study has examined the effects of a COX10 mutation on OA risk. COX10 missense mutation is associated with mitochondrial dysfunction. In the present study, the participants with the minor COX10_rs62054459 allele were inversely associated with OA risk. COX10 gene expression in the articular cartilage were not provided according to COX10_rs62054459. However, because OA is related to skeletal muscle and adipose tissues, their COX10 expression levels were determined. Interestingly, COX10 expression in people with the rs62054459 minor allele had higher expression in the skeletal muscle, subcutaneous adipose tissue, and visceral adipose tissue in eQTL analysis. This suggests that people with the rs62054459 minor allele might have better mitochondrial function. Therefore, people with the COX10 rs62054459 minor allele might have a protective effect in OA by increasing COX10 expression.

The association of AIGI with OA has not been studied. AIGI acts as a hydrolase of fatty acid esters of hydroxy fatty acids located in the integral membrane, but not other lipids [30]. It is expressed at higher levels in the heart, ovary, testes, liver, kidney, small intestine, and colon and lower levels in the spleen, prostate, brain, skeletal muscle, and pancreas. AIG1 expression in articular cartilage has not been reported. The present study showed that AIG1 expression was lower in those with the minor allele AIG1_rs6570550, than those with the major allele in eQTL analysis. Furthermore, the AIGI genetic variant effect may be involved in gender differences in OA incidence, as women have a much higher incidence of OA than men. Decreased AIG1 expression, due to having the minor allele AIG1_rs6570550, might be associated with OA risk.

OA risk is affected by lifestyle, but few regimes efficiently prevent or reduce the it [31]. Fish oil, protein, dietary fiber, and polyphenols, including curcumin, are recommended to improve OA symptoms [32-35]. On the other hand, their efficacy is varied and controversial [36]. OA risk is closely related to obesity, and weight loss is recommended for reducing 
OA symptoms. Intermittent fasting is one weight-loss method and OA therapy [33]. The present study showed that PRS interacted with dietary intake, including energy, protein, fat, and alcohol, to influence OA risk, even though dietary intake did not affect OA risk. In the present study, adults with high-PRS demonstrated a higher OA risk with high-energy intake and low protein and fat intake. On the other hand, dietary patterns did not significantly affect OA risk. Therefore, adults aged $>40$ years might need a lower energy intake with nutritious foods, including high protein, and maintain body weight within a normal range to prevent or alleviate OA symptoms.

Polygenetic variants including COX10 and AIG1 were associated with OA risk, and the impact of the two genetic variants on OA was related to their gene expression. As COX10 and AIG1 are involved in energy and sex hormone metabolism, respectively, the present study indirectly demonstrated the genetic impact of the energy metabolism and gender relationship in OA risk. The present study demonstrated that BMI, waist circumference, and fat mass, but not lean body mass, were positively associated with OA risk, and PRS and energy intake had an interaction showing that low energy intake decreased OA risk primarily in participants with high-PRS. Genetic variants of COX10 and AIG1 were indirectly involved in OA risk through modulating energy metabolism. Additionally, the minor-alleles of COX-10 modulated the COX-10 protein expression to influence OA risk. Therefore, genetic variants involved in energy, inflammation, and neuronal networks are associated with OA risk.

The present study is the first to determine genetic variants to show that PRS-PRS and PRS-lifestyle interactions influence OA risk. Nevertheless, the present study had some limitations. First, this was a case-control study to collect data cross-sectionally. Second, OA incidence was reported by a questionnaire of an OA diagnosis by a physician, even though the rheumatoid arthritis incidence was asked separately. Third, gene expression according to genetic variant alleles was not determined in the articular cartilage. Finally, lifestyle and nutrient intake were self-reported with questionnaires and may have included some memory biases.

In conclusion, PRS generated from the five-SNP best model with SNP-SNP interaction for OA risk included AIG1_rs6570550, COX10_rs62054459,DLG2_rs148643344, PLXNA4_ rs1472529430, and SOX5_rs73283615. After adjusting for the covariates related to OA risk, the high-PRS participants were 3.89 times more positively associated with OA risk. The PRS did not have an association with metabolic syndrome and insulin resistance. Energy, protein, and fat intake interacted with PRS to influence OA risk. In the high energy, low protein, and low-fat intake groups, the PRS impact on OA incidence was much higher than in the low intake group. In taking together nutrient intake, the high-WSD lowered OA incidence more than the low-WSD in contrast to metabolic syndrome risk. Therefore, adults with a high-PRS should consume less energy than EER and have a high protein and moderate fat intake to prevent or alleviate OA symptoms. The PRS data can be applied to personalized nutrition for preventing $\mathrm{OA}$.

Supplementary Materials: The following supporting information can be downloaded at: https: / / www.mdpi.com/article/10.3390/diagnostics12020340/s1. Table S1: Factor loadings of food groups in dietary patterns identified using principal component analysis. Table S2: The characteristics of the ten genetic variants of genes related to osteoarthritis $(\mathrm{OA})$ in adults.

Funding: This research was supported by Hoseo University (20200817).

Institutional Review Board Statement: The study was conducted according to the guidelines of the Declaration of Helsinki and approved by the Institutional Review Boards of the Korean National Institute of Health (1041231-190902-BR-099-01) and Hoseo University (1041231-150811-HR-034-01) on 24 March 2019.

Informed Consent Statement: Written informed consent was obtained from all participants.

Data Availability Statement: The data that support the findings of this study are available from the corresponding author upon reasonable request. 
Conflicts of Interest: There are no conflict of interest.

\section{References}

1. Fransen, M.; Bridgett, L.; March, L.; Hoy, D.; Penserga, E.; Brooks, P. The epidemiology of osteoarthritis in Asia. Int. J. Rheum. Dis. 2011, 14, 113-121. [CrossRef] [PubMed]

2. Yucesoy, B.; Charles, L.E.; Baker, B.; Burchfiel, C.M. Occupational and genetic risk factors for osteoarthritis: A review. Work 2015, 50, 261-273. [CrossRef] [PubMed]

3. Alissa, E.M.; Alzughaibi, L.S.; Marzouki, Z.M. Dietary intake of fatty acids and antioxidants in relation to radiographic knee osteoarthritis: Results from a case-control study. J. Hum. Nutr. Diet 2020, 33, 431-438. [CrossRef]

4. Coskun Benlidayi, I. Diet in osteoarthritis. Rheumatol. Int. 2021, 41, 1699-1700. [CrossRef] [PubMed]

5. Allen, K.D. Racial and ethnic disparities in osteoarthritis phenotypes. Curr. Opin. Rheumatol. 2010, $22,528-532$. [CrossRef] [PubMed]

6. $\quad$ Loughlin, J.; Mustafa, Z.; Smith, A.; Irven, C.; Carr, A.J.; Clipsham, K.; Chitnavis, J.; Bloomfield, V.A.; McCartney, M.; Cox, O.; et al. Linkage analysis of chromosome $2 q$ in osteoarthritis. Rheumatology 2000, 39, 377-381. [CrossRef]

7. Xu, P.; Yao, J.; Hou, W. Relationships between COL2A1 gene polymorphisms and knee osteoarthritis in Han Chinese women. Mol. Biol Rep. 2011, 38, 2377-2381. [CrossRef]

8. De Sire, A.; de Sire, R.; Petito, V.; Masi, L.; Cisari, C.; Gasbarrini, A.; Scaldaferri, F.; Invernizzi, M. Gut-joint axis: The role of physical exercise on gut microbiota modulation in older people with osteoarthritis. Nutrients 2020, 12, 574. [CrossRef]

9. Hong, K.W.; Oh, B. Recapitulation of genome-wide association studies on body mass index in the Korean population. Int. J. Obes. 2012, 36, 1127-1130. [CrossRef]

10. Bedogni, G.; Malavolti, M.; Severi, S.; Poli, M.; Mussi, C.; Fantuzzi, A.L.; Battistini, N. Accuracy of an eight-point tactile-electrode impedance method in the assessment of total body water. Eur. J. Clin. Nutr. 2002, 56, 1143-1148. [CrossRef]

11. Park, S.; Ahn, J.; Lee, B.K. Self-rated subjective health status is strongly associated with sociodemographic factors, lifestyle, nutrient intakes, and biochemical indices, but not smoking status: KNHANES 2007-2012. J. Korean Med. Sci. 2015, 30, 1279-1287. [CrossRef] [PubMed]

12. Kim, Y.; Han, B.G. Cohort profile: The Korean genome and epidemiology study (KoGES) consortium. Int. J. Epidemiol. 2017, 46, 1350. [CrossRef] [PubMed]

13. Seo, M.H.; Lee, W.-Y.; Kim, S.S.; Kang, J.-H.; Kang, J.-H.; Kim, K.K.; Kim, B.-Y.; Kim, Y.-H.; Kim, W.-J.; Kim, E.M.; et al. 2018 Korean society for the study of obesity guideline for the management of obesity in Korea. J. Obes. Metab. Syndr. 2019, 28, 40-45. [CrossRef] [PubMed]

14. Ahn, Y.; Kwon, E.; Shim, J.E.; Park, M.K.; Joo, Y.; Kimm, K.; Park, C.; Kim, D.H. Validation and reproducibility of food frequency questionnaire for Korean genome epidemiologic study. Eur. J. Clin. Nutr. 2007, 61, 1435-1441. [CrossRef] [PubMed]

15. Ahn, Y.L.J.; Cho, N.H.; Shin, C.; Park, C.; Oh, B.S.; Kimm, K. Validation and calibration of semi-quantitative food frequency questionnaire-With participants of the Korean health and genome study. Korean J. Community Nutr. 2004, 9, $173-182$.

16. Paik, H.Y. Dietary reference intakes for Koreans (KDRIs). Asia Pac. J. Clin. Nutr. 2008, 17 (Suppl. 2), 416-419.

17. Park, S.; Kang, S. A Western-style diet interacts with genetic variants of the LDL receptor to hyper-LDL cholesterolemia in Korean adults. Public Health Nutr. 2020, 24, 2964-2974. [CrossRef]

18. Rabbee, N.; Speed, T.P. A genotype calling algorithm for affymetrix SNP arrays. Bioinformatics 2006, 22, 7-12. [CrossRef]

19. Uma Jyothi, K.; Reddy, B.M. Gene-gene and gene-environment interactions in the etiology of type 2 diabetes mellitus in the population of Hyderabad, India. Meta Gene 2015, 5, 9-20. [CrossRef] [PubMed]

20. Khera, A.V.; Chaffin, M.; Wade, K.H.; Zahid, S.; Brancale, J.; Xia, R.; Distefano, M.; Senol-Cosar, O.; Haas, M.E.; Bick, A.; et al Polygenic prediction of weight and obesity trajectories from birth to adulthood. Cell 2019, 177, 587-596. [CrossRef]

21. Lewis, C.M.; Vassos, E. Polygenic risk scores: From research tools to clinical instruments. Genome Med. 2020, 12, 44 [CrossRef] [PubMed]

22. Zhou, J.-Y.; Song, M.Y.; Park, S. Carbohydrate and sodium intake and physical activity interact with genetic risk scores of four genetic variants mainly related to lipid metabolism to modulate metabolic syndrome risk in Korean middle-aged adults. $\mathrm{Br}$. $\mathrm{J}$. Nutr. 2019, 122, 919-927. [CrossRef] [PubMed]

23. Bateman, J.F. Genetic aspects of osteoarthritis. Semin. Arthritis Rheum. 2005, 34, 15-18. [CrossRef] [PubMed]

24. Gari, M.A.; AlKaff, M.; Alsehli, H.S.; Dallol, A.; Gari, A.; Abu-Elmagd, M.; Kadam, R.; Abuzinadah, M.F.; Gari, M.; Abuzenadah, A.M.; et al. Identification of novel genetic variations affecting osteoarthritis patients. BMC Med. Genet. 2016, 17, 68. [CrossRef] [PubMed]

25. Mabey, T.; Honsawek, S. Cytokines as biochemical markers for knee osteoarthritis. World J. Orthoped. 2015, 6, 95-105. [CrossRef]

26. Tait Wojno, E.D.; Hunter, C.A.; Stumhofer, J.S. The immunobiology of the interleukin-12 family: Room for discovery. Immunity 2019, 50, 851-870. [CrossRef]

27. Nees, T.A.; Rosshirt, N.; Zhang, J.A.; Reiner, T.; Sorbi, R.; Tripel, E.; Walker, T.; Schiltenwolf, M.; Hagmann, S.; Moradi, B. Synovial cytokines significantly correlate with osteoarthritis-related knee pain and disability: Inflammatory mediators of potential clinical relevance. J. Clin. Med. 2019, 8, 1343. [CrossRef]

28. Xu, J.; Kang, Y.; Liao, W.-M.; Yu, L. MiR-194 Regulates chondrogenic differentiation of human adipose-derived stem cells by targeting Sox5. PLoS ONE 2012, 7, e31861. [CrossRef] 
29. Diaz, F.; Thomas, C.K.; Garcia, S.; Hernandez, D.; Moraes, C.T. Mice lacking COX10 in skeletal muscle recapitulate the phenotype of progressive mitochondrial myopathies associated with cytochrome c oxidase deficiency. Hum. Mol. Genet. 2005, 14, 2737-2748. [CrossRef]

30. Parsons, W.H.; Kolar, M.J.; Kamat, S.S.; Iii, A.B.C.; Hulce, J.J.; Saez, E.; Kahn, B.B.; Saghatelian, A.; Cravatt, B.F. AIG1 and ADTRP are atypical integral membrane hydrolases that degrade bioactive FAHFAs. Nat. Chem. Biol. 2016, 12, 367-372. [CrossRef]

31. Baumbach, L.; Roos, E.M.; Lykkegaard, J.; Thomsen, K.S.; Kristensen, P.L.; Christensen, A.I.; Thorlund, J.B. Patients with osteoarthritis are least likely to receive lifestyle advice compared with patients with diabetes and hypertension: A national health survey study from Denmark. Osteoarthr. Cartil. Open 2020, 2, 100067. [CrossRef]

32. Valsamidou, E.; Gioxari, A.; Amerikanou, C.; Zoumpoulakis, P.; Skarpas, G.; Kaliora, A.C. Dietary interventions with polyphenols in osteoarthritis: A systematic review directed from the preclinical data to randomized clinical studies. Nutrients 2021, 13, 1420. [CrossRef] [PubMed]

33. Park, S.; Shin, B.K. Intermittent fasting with a high-protein diet mitigated osteoarthritis symptoms by increasing lean body mass and reducing inflammation in osteoarthritic rats with Alzheimer's disease-like dementia. Br. J. Nutr. 2021, 127, 55-67. [CrossRef] [PubMed]

34. Daily, J.W.; Yang, M.; Park, S. Efficacy of turmeric extracts and curcumin for alleviating the symptoms of joint arthritis: A systematic review and meta-analysis of randomized clinical trials. J. Med. Food 2016, 19, 717-729. [CrossRef] [PubMed]

35. Kuszewski, J.C.; Wong, R.H.X.; Howe, P.R.C. Fish oil supplementation reduces osteoarthritis-specific pain in older adults with overweight/obesity. Rheumatol. Adv. Pract. 2020, 4, rkaa036. [CrossRef]

36. MacFarlane, L.A.; Cook, N.R.; Kim, E.; Lee, I.M.; Iversen, M.D.; Gordon, D.; Buring, J.E.; Katz, J.N.; Manson, J.E.; Costenbader, K.H. The effects of vitamin D and marine omega-3 fatty acid supplementation on chronic knee pain in older US adults: Results from a randomized trial. Arthritis Rheumatol. 2020, 72, 1836-1844. [CrossRef] [PubMed] 